\title{
Risk Factors of Geriatric Syndromes in Korean Population
}

\author{
Sunyoung Kim², Jong-heon Park', Hongyup $\mathrm{Ahn}^{3}$, Sohee Lee ${ }^{3}$, Hyung Joon Yoo, Jinho Yoo ${ }^{5}$, Chang Won Won ${ }^{1,5}$, \\ Geriatric Syndromes Study Group of Korea Geriatrics Society \\ ${ }^{1}$ Department of Medicine, Graduate School, Kyung Hee University, Seoul, ${ }^{2}$ Big Data Steering Department, National Health Insurance Service, \\ Wonju, ${ }^{3}$ Department of Statistics, Dongguk University, Seoul, ${ }^{4}$ Division of Endocrinology \& Geriatrics, Department of Internal Medicine, \\ Hallym University College of Medicine, Seoul, ${ }^{5}$ Department of Family Medicine, Kyung Hee University Medical Center, Seoul, Korea
}

Corresponding Author: Chang Won Won, MD, PhD Department of Family Medicine, Kyung Hee University Medical Center, 23 Kyungheedae-ro, Dongdaemun-gu, Seoul 02447, Korea

Tel: $+82-2-958-8697$ Fax: +82-2-958-8699

E-mail: chunwon62@naver.com
Background: This study was conducted to identify the risk factors of geriatric syndromes including fall-related fractures, delirium, incontinence, and pressure ulcers using the Korean nationwide claims data. Methods: The claims data of the Korean National Health Insurance Service between 2006 and 2015 were used. We included older people, 65 years of age and older, who underwent a national health check-up in 2007 or 2008. Geriatric syndromes were limited to 4 types (fall-related fractures, delirium, incontinence, and pressure ulcers), which can be identified via diagnostic codes. Results: The risks of fall-related fractures, delirium, incontinence, and pressure ulcers all increased with increasing age, and the risks of delirium and incontinence increased more in women than in men. Exercise decreased the risks of all 4 types of geriatric syndrome, and increase in body mass index decreased the risks of fall-related fractures, delirium, and pressure ulcers while increasing the risk of incontinence. The adjusted hazard ratios of smoking and alcohol intake three times or more a week in relation to fall-related fractures, delirium, incontinence, and pressure ulcers were 1.46, 1.16, 0.99, and 1.33, respectively, and 1.04, 1.12, 0.93, 0.98, respectively. Conclusion: This study showed that increasing age, major surgery within 6 months, and polypharmacy increase the risk of 4 types of geriatric syndrome in Korea. Comorbidity (especially dementia) was identified as a very important risk factor. Exercise had protective effects against the 4 types of geriatric syndrome studied. However, contradictory results were found for smoking and alcohol intake, which require further study.

Key Words: Fall, Delirium, Incontinence, Pressure Ulcer, National Health Insurance
Received: June 19, 2017

Revised: August 1, 2017

Accepted: August 12, 2017 pressure ulcers, falls, delirium, frailty, and dementia and is usually manifested by a single symptom due to the interaction of pathophysiologies of multiple causes unlike other syndromes ${ }^{4,5)}$. Geriatric syndrome incurs disabling features in everyday life, thereby decreasing the quality of life in old age and increasing mortality in older people ${ }^{6)}$.

There is a growing interest in geriatric syndrome in the medical, governmental, and public fields, raising the necessity for understanding, systematic management of, and preventive measures for geriatric syndrome. Therefore, it is important to identify the current situation and relevant risk factors in order to build the foundation for management. In Korea, most of the population is enrolled in the National Health Insurance Service (NHIS), which provides the sole national claims database and the requisites for studying various diseases and their burdens ${ }^{7)}$.

Consequently, we aimed to identify the essential information required for systematic management of geriatric syndromes, including falls, delirium, incontinence, and frailty, by 
analyzing relevant risk factors using the health insurance data.

\section{MATERIALS AND METHODS}

\section{Data Sources}

South Korea has a universal health coverage system, the NHIS, which covers approximately $98 \%$ of the Korean population. The NHIS is responsible for the payment of medical expenses to providers such as medical, check-up, and longterm care institutions that manage the qualifications and insurance premiums of the subscribers and provide medical care, examinations, and nursing services. As a result, the NHIS has been providing medical services including diagnosis, treatment, procedures, surgical history, and prescription drugs; qualification and insurance premiums; cancer registration information; medical benefit data; and health checkup results for 14 years and has accumulated more than one trillion data during this period. Therefore, these data contain the representative and comprehensive characteristics of the medical services of the whole nation ${ }^{7)}$.

We used the medical claims data of the NHIS from January 2002 to December 2015. In 2007, there were 4,851,643 individuals aged 65 and older who were covered by the NHIS. Further, of $1,678,458$ of these patients who underwent a national health check-up from 2007 to 2008, 1,350,961 never had any of the 4 diagnostic codes for geriatric syndromes between 2002 and 2006 and were screened for the diagnosis of geriatric syndrome from 2007 to 2015 (Figs. 1, 2). The main and subsidiary diagnostic codes of inpatients and outpatients of primary, secondary, and tertiary medical institutions other than dental and oriental hospitals were evaluated for fall-related fractures, delirium, incontinence, pressure ulcers, and frailty among other geriatric syndromes.

\section{Study Design}

The NHIS database was used to identify the prevalence of geriatric syndrome. The main and subsidiary diagnostic codes of fracture of the femur (S72.0), fracture of the lower end of the radius (S52.5), and fractures of the spine (S22-32) that often result from falls were defined as fall-related fractures. The codes of delirium not induced by alcohol and other psychoactive substances (F05), delirium not superimposed on dementia (F05.0, F05.1), other delirium (F05.8), and delirium unspecified (F05.9) were used to define delirium. The codes of N39.3, N39.4, F98.1, F98.5, R15, and R32 were used to define incontinence, and the code of L89 was used to define pressure ulcers.

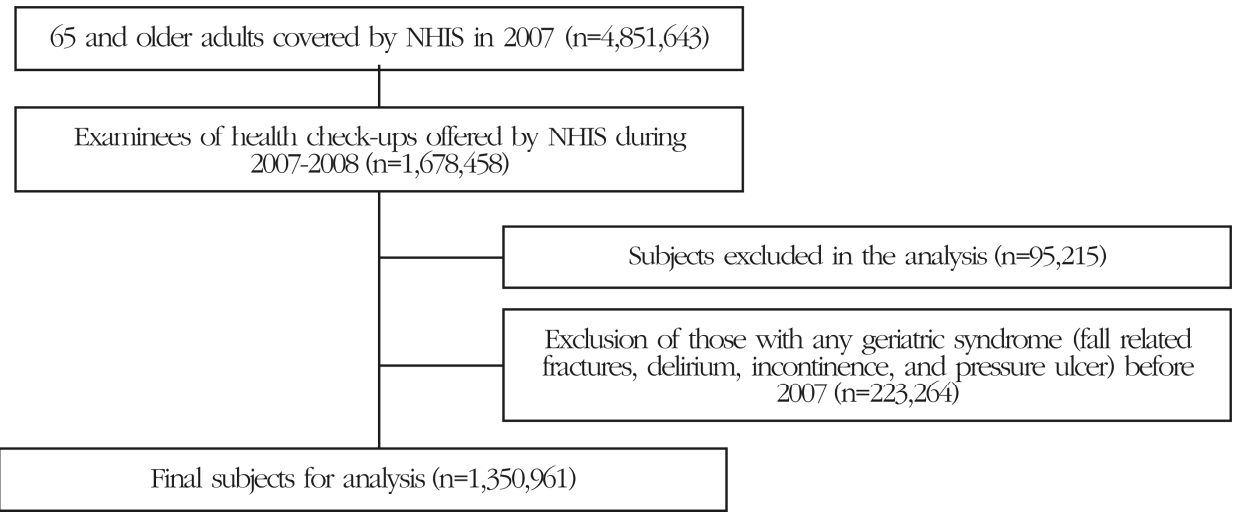

Fig. 1. Patient flow chart. NHIS, National Health Insurance Service.

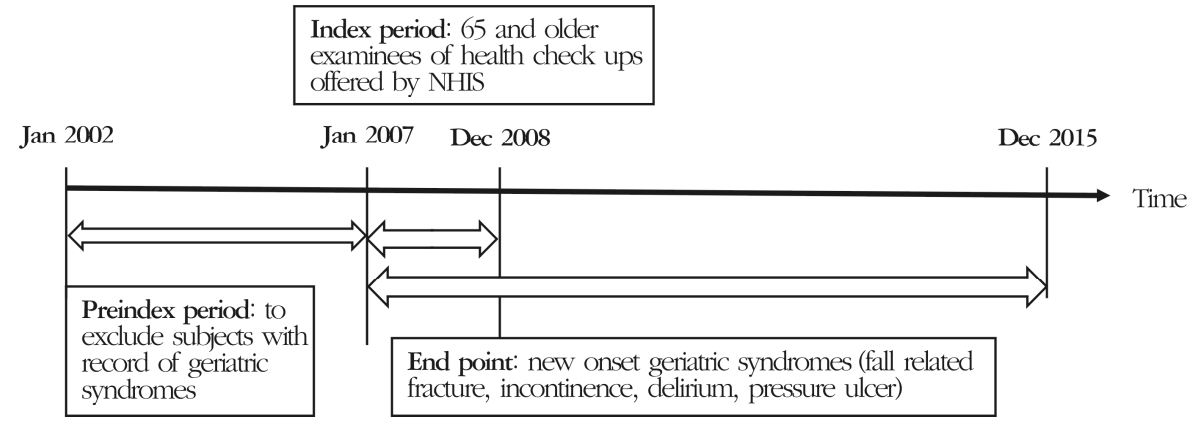

Fig. 2. Study design. NHIS, National Health Insurance Service. 


\section{Covariate Data}

Medical prescriptions (number of drugs and number of days per prescription), major surgery within the last 6 months, and comorbidities were evaluated using the claims data. Comorbidities were evaluated using the most widely used assessment tool, the Charlson comorbidity index. Specific weighted scores ranging from 0 to 6 were assigned to the main or subsidiary diagnoses, and the sum of the weighted scores was used for adjustment ${ }^{8)}$. Diagnostic codes in the main and subsidiary diagnoses were used for each condition. Major surgeries including cataract surgery, percutaneous coronary interventions (percutaneous transluminal coronary angioplasty and stenting), coronary bypass, ligation/stripping of varicose veins, and appendectomy ${ }^{9)}$ were included if the surgical codes were entered within 6 months of the diagnostic codes of geriatric syndrome. Polypharmacy was defined as five or more of drugs prescribed for at least $80 \%$ of the year, using the main components of the prescribed drugs as the standards for comparison.

Health check-up data were also used to identify the risk factors of geriatric syndrome. Variables of interest included age, body mass index (BMI, for low weight and obesity), history of chronic diseases (diabetes mellitus, hypertension, and dyslipidemia), lifestyle (smoking, drinking, and exercise), and blood tests for hemoglobin and total cholesterol levels.

\section{Ethics Statement}

This study was approved by the Kyung Hee University Hospital Research Ethics Committee, and did not require written informed consent form since the NHIS provided coded data without any confidential information of patients (approval number: KMC IRB 1522-08).

\section{Statistical Analysis}

Baseline characteristics are presented as a mean with standard error of the mean or numbers and percentages. Cox hazard regression analysis was used to evaluate the association between risk factors including the health-related behaviors (smoking and physical activity) and geriatric syndromes. Statistical analyses were performed using SAS ver. 9.2 (SAS Institute Inc., Cary, NC, USA). A p-value $<0.05$ was considered statistically significant.

\section{RESULTS}

\section{Geriatric Syndrome and Associated Factors}

The mean ages of patients with incident geriatric syndromes were $73.5,72.4,71.8$, and 73.8 years for fall-related fractures, delirium, incontinence, and pressure ulcers, respec- tively. These patients were older than those in the noninflicted groups. The proportion of men was higher in patients with fall-related fractures (57.16\%) and pressure ulcers (58.38\%). The incidence rate of all four geriatric syndromes was higher in non-urban areas than in urban areas. The rate of obesity was higher only in the incontinence group as compared with the noninflicted group. The proportion of current smokers and patients with alcohol intake 3 or more times a week was higher in the fall-related fracture and pressure ulcer groups than in the other groups. The proportion of people engaging in regular exercise was lower, and that of people in the lower 20\% income group was higher, in the groups of fall-related fractures, delirium, incontinence, and pressure ulcers. All geriatric syndrome groups had higher rates of comorbidities, major surgery within the last 6 months, and polypharmacy. The hemoglobin level was lower in all geriatric syndrome groups (Table 1).

\section{Incidence and Hazard Ratio of Geriatric Syndrome According to Age and Sex}

The incidence rates, in 1,000 person-years, of fall-related fractures, delirium, incontinence, and pressure ulcers were $4.37,27.67,13.61$, and 9.45 , respectively. The risks of fall-related fractures, delirium, incontinence, and pressure ulcers all increased with increasing age. Age was categorized intro three groups, 65-69, 70-74, and 85 years and above, and patients 85 years old and older had 3.2 times higher risk of fall-related fractures and 3.5 times higher risk of pressure ulcers than patients 65-69 years old. Women had 2.39 times higher risk of delirium and 2.37 times higher risk of incontinence than men (Table 2).

Smoking was related to a higher risk of fall-related fractures, delirium, and pressure ulcers. The risks of fall-related fractures and delirium were higher in people drinking alcohol three or more times a week than in those who do not. Physical activity had preventive effects on fall-related fractures, delirium, incontinence, and pressure ulcers. Low body weight increased the risks of fall-related fractures, delirium, and pressure ulcers by $1.2,1.3$, and 1.4 times, respectively, compared with the normal BMI group, and only the risk of incontinence increased with higher BMI. Hypertension decreased the risk of delirium, but all other acute and chronic diseases investigated increased the risks of fall-related fractures, delirium, incontinence, and pressure ulcers. Polypharmacy and major surgery in past 6 months were related to higher risks of all four types of geriatric syndrome (Table 3 ).

\section{Adjusted Hazard Ratio of Risk Factors of Geriatric Syndrome}

Stepwise scoring of the major risk factors of geriatric syndrome identified in Table 2 revealed that old age, low 
$\mathrm{BMI}$, history of major surgery within the past 6 months, comorbidities, and polypharmacy all increased the risks of fallrelated fractures, delirium, incontinence, and pressure ulcers (Supplementary Table 1).

\section{DISCUSSION}

Geriatric syndrome induces commonly serious conditions in older people that gravely affect their function and quality of life. Geriatric syndromes are caused by various risk factors and require integrated care ${ }^{10)}$. It has been suggested that treating one geriatric syndrome diagnosed in an inpatient or an outpatient may serve to manage other geriatric syndromes as well, since they commonly share fundamental risk factors. Inoue et al. reported that general geriatric syndromes such as pressure ulcers, incontinence, delirium, falls, and functional decline all share the risk factors of old age, cognitive disorder, functional disorder, and mobility disorder ${ }^{4}$.

The most important finding of this study is that fall-related fractures, delirium, incontinence, and pressure ulcers all have the common risk factors of old age, history of major surgery within the past 6 months, and polypharmacy. Regarding sex

Table 1. Baseline characteristics of people with each geriatric syndrome

\begin{tabular}{|c|c|c|c|c|c|c|c|c|}
\hline \multirow{2}{*}{ Characteristic } & \multicolumn{2}{|c|}{ Fall-related fracture } & \multicolumn{2}{|c|}{ Delirium } & \multicolumn{2}{|c|}{ Incontinence } & \multicolumn{2}{|c|}{ Pressure ulcer } \\
\hline & No & Yes & No & Yes & No & Yes & No & Yes \\
\hline Total number & $1,307,568$ & 43,393 & $1,098,760$ & 252,201 & $1,221,099$ & 129,862 & $1,257,199$ & 93,762 \\
\hline Age (yr) & $71.5 \pm 4.5$ & $73.5 \pm 5$ & $71.3 \pm 4.4$ & $72.4 \pm 4.8$ & $71.5 \pm 4.5$ & $71.8 \pm 4.4$ & $71.4 \pm 4.4$ & $73.8 \pm 5.3$ \\
\hline Male sex & $639,184(48.88)$ & $24,803(57.16)$ & $590,765(53.77)$ & $73,222(29.03)$ & $627,099(51.36)$ & $36,888(28.41)$ & $609,253(48.46)$ & $54,734(58.38)$ \\
\hline Place, rural & $801,824(61.33)$ & 27,035 (62.31) & $666,008(60.62)$ & $162,851(64.58)$ & 745,318 (61.04) & $83,541(64.34)$ & $768,425(61.13)$ & 60,434 (64.46) \\
\hline Smoking & $162,009(12.73)$ & 7,585 (17.94) & 145,466 (13.6) & $24,128(9.85)$ & $159,536(13.42)$ & 10,058 (7.98) & 154,045 (12.59) & $15,549(17.05)$ \\
\hline \multicolumn{9}{|l|}{ Alcohol intake } \\
\hline Nondrinker & $953,189(74.48)$ & $31,190(73.47)$ & $782,056(72.69)$ & $202,323(82.14)$ & $879,078(73.53)$ & 105,301 (83.12) & $916,122(74.44)$ & $68,257(74.5)$ \\
\hline$\leq 2 / \mathrm{wk}$ & $196,623(15.36)$ & $6,026(14.2)$ & $176,416(16.4)$ & $26,233(10.65)$ & 188,717 (15.79) & $13,932(11)$ & $190,167(15.45)$ & $12,482(13.62)$ \\
\hline$\geq 3 / \mathrm{wk}$ & $129,957(10.15)$ & $5,235(12.33)$ & $117,432(10.91)$ & $17,760(7.21)$ & $127,745(10.69)$ & $7,447(5.88)$ & $124,317(10.1)$ & 10,875 (11.87) \\
\hline Exercise & $502,852(39.35)$ & 14,246 (33.66) & $438,186(40.79)$ & $78,912(32.08)$ & $472,790(39.61)$ & $44,308(34.98)$ & 487,707 (39.69) & $29,391(32.2)$ \\
\hline Income, low & 210,369 (16.31) & 6,382 (14.92) & 177,247 (16.35) & $39,504(15.89)$ & $196,738(16.33)$ & $20,013(15.64)$ & $202,364(16.32)$ & $14,387(15.56)$ \\
\hline $\mathrm{BMI}\left(\mathrm{kg} / \mathrm{m}^{2}\right)$ & $23.7 \pm 3.2$ & $23.3 \pm 3.2$ & $23.8 \pm 3.1$ & $23.5 \pm 3.3$ & $23.7 \pm 3.1$ & $24.3 \pm 3.3$ & $23.8 \pm 3.1$ & $23.2 \pm 3.3$ \\
\hline CCI & $1.7 \pm 1.8$ & $2.3 \pm 2.1$ & $1.7 \pm 1.8$ & $1.8 \pm 1.8$ & $1.7 \pm 1.8$ & $2.1 \pm 1.9$ & $1.7 \pm 1.7$ & $2.3 \pm 2.2$ \\
\hline \multicolumn{9}{|l|}{ Comorbidities } \\
\hline Hypertension & $773,677(59.17)$ & 27,538 (63.46) & $653,082(59.44)$ & $148,133(58.74)$ & $720,128(58.97)$ & 81,087 (62.44) & $740,980(58.94)$ & 60,235 (64.24) \\
\hline Dyslipidemia & $314,505(24.05)$ & $10,586(24.4)$ & $261,980(23.84)$ & $63,111(25.02)$ & $288,466(23.62)$ & $36,625(28.2)$ & $303,450(24.14)$ & $21,641(23.08)$ \\
\hline Diabetes mellitus & $319,590(24.44)$ & $13,899(32.03)$ & $270,955(24.66)$ & $62,534(24.8)$ & 295,671 (24.21) & 37,818 (29.12) & $301,938(24.02)$ & $31,551(33.65)$ \\
\hline Stroke & 175,113 (13.39) & 8,807 (20.3) & 145,171 (13.21) & $38,749(15.36)$ & $161,598(13.23)$ & $22,322(17.19)$ & $163,928(13.04)$ & $19,992(21.32)$ \\
\hline Dementia & 28,205 (2.16) & $2,470(5.69)$ & $23,321(2.12)$ & 7,354 (2.92) & $26,589(2.18)$ & $4,086(3.15)$ & $24,889(1.98)$ & $5,786(6.17)$ \\
\hline Heart disease & $92,421(7.07)$ & 4,755 (10.96) & $76,360(6.95)$ & $20,816(8.25)$ & 85,750 (7.02) & $11,426(8.8)$ & $86,649(6.89)$ & $10,527(11.23)$ \\
\hline Pulmonary disease & $288,264(22.05)$ & 12,211 (28.14) & $239,129(21.76)$ & $61,346(24.32)$ & $266,794(21.85)$ & 33,681 (25.94) & $274,826(21.86)$ & $25,649(27.36)$ \\
\hline Liver disease & $48,002(3.67)$ & 2,155 (4.97) & $40,452(3.68)$ & $9,705(3.85)$ & $44,821(3.67)$ & $5,336(4.11)$ & $45,356(3.61)$ & $4,801(5.12)$ \\
\hline Kidney disease & $12,441(0.95)$ & $1,000(2.3)$ & 10,955 (1) & $2,486(0.99)$ & $12,282(1.01)$ & $1,159(0.89)$ & $11,543(0.92)$ & $1,898(2.02)$ \\
\hline Cancer & $78,726(6.02)$ & 3,594 (8.28) & $68,346(6.22)$ & $13,974(5.54)$ & $74,188(6.08)$ & $8,132(6.26)$ & $73,792(5.87)$ & $8,528(9.1)$ \\
\hline Osteoporosis & $218,305(16.7)$ & 7,483 (17.24) & $162,578(14.8)$ & $63,210(25.06)$ & $191,562(15.69)$ & $34,226(26.36)$ & $210,130(16.71)$ & $15,658(16.7)$ \\
\hline Urinary tract infection & $124,916(9.55)$ & $4,777(11.01)$ & 99,919 (9.09) & $29,774(11.81)$ & $104,769(8.58)$ & 24,924 (19.19) & $119,212(9.48)$ & $10,481(11.18)$ \\
\hline Pneumonia & $61,871(4.73)$ & $3,080(7.1)$ & $51,209(4.66)$ & $13,742(5.45)$ & $58,079(4.76)$ & 6,872 (5.29) & $57,863(4.6)$ & $7,088(7.56)$ \\
\hline Tuberculosis & $34,850(2.67)$ & $1,699(3.92)$ & $29,109(2.65)$ & $7,440(2.95)$ & $32,569(2.67)$ & $3,980(3.06)$ & $32,765(2.61)$ & $3,784(4.04)$ \\
\hline Major surgery & $42,609(3.26)$ & $1,718(3.96)$ & $34,636(3.15)$ & $9,691(3.84)$ & 38,897 (3.19) & $5,430(4.18)$ & $40,688(3.24)$ & $3,639(3.88)$ \\
\hline Medication & $1.4 \pm 2.1$ & $1.8 \pm 2.5$ & $1.4 \pm 2.1$ & $1.4 \pm 2.1$ & $1.4 \pm 2.1$ & $1.6 \pm 2.2$ & $1.4 \pm 2.1$ & $1.9 \pm 2.5$ \\
\hline Polypharmacy & $129,557(9.91)$ & 6,558 (15.11) & $110,396(10.05)$ & $25,719(10.2)$ & $120,997(9.91)$ & 15,118 (11.64) & $121,536(9.67)$ & $14,579(15.55)$ \\
\hline Hemoglobin (g/dL) & $13.4 \pm 1.4$ & $13.3 \pm 1.5$ & $13.5 \pm 1.4$ & $13 \pm 1.4$ & $13.4 \pm 1.5$ & $13.1 \pm 1.4$ & $13.4 \pm 1.4$ & $13.3 \pm 1.6$ \\
\hline Total cholesterol (mg/dL) & $196.6 \pm 38.3$ & $193.6 \pm 40$ & $196 \pm 38.2$ & $198.8 \pm 39.1$ & $196.1 \pm 38.3$ & $200.3 \pm 39.1$ & $196.8 \pm 38.3$ & $192.6 \pm 39.9$ \\
\hline
\end{tabular}

Values are presented as mean \pm standard deviation or number (\%).

BMI, body mass index; CCI, Charlson comorbidity index; TB, tuberculosis.

All p-values $<0.001$. p-values obtained by t-test and chi-square test.

Major surgery refers to people who underwent a major surgery within the past 6 months; medication refers to the mean number of prescription drugs; polypharmacy was defined as $\geq 5$ medications/day; and income (low) refers to the lowest quartile of income. 
Table 2. Incidence rates of geriatric syndromes and hazard ratios by age and sex

\begin{tabular}{|c|c|c|c|c|c|c|c|c|c|c|c|c|}
\hline \multirow[b]{2}{*}{ Variable } & \multicolumn{3}{|c|}{ Fall-related fracture } & \multicolumn{3}{|c|}{ Delirium } & \multicolumn{3}{|c|}{ Incontinence } & \multicolumn{3}{|c|}{ Pressure ulcer } \\
\hline & Event & $\begin{array}{l}\text { IR (per } \\
1,000)\end{array}$ & $\mathrm{HR}(95 \% \mathrm{C})$ & Event & $\begin{array}{l}\text { IR (per } \\
1,000)\end{array}$ & $\mathrm{HR}(95 \% \mathrm{C})$ & Event & $\begin{array}{l}\text { IR (per } \\
1,000)\end{array}$ & HR (95\% C) & Event & $\begin{array}{l}\text { IR (per } \\
1,000)\end{array}$ & $\mathrm{HR}(95 \% \mathrm{C})$ \\
\hline Total number & 43,393 & 4.372 & & 252,201 & 27.669 & & 129,862 & 13.617 & & 93,762 & 9.485 & \\
\hline \multicolumn{13}{|l|}{ Age (yr) } \\
\hline $65-69$ & 11,961 & 2.644 & $\begin{array}{c}1 \\
\text { (reference) }\end{array}$ & 90,854 & 21.553 & $\begin{array}{c}1 \\
\text { (reference) }\end{array}$ & 52,309 & 12.011 & $\begin{array}{c}1 \\
\text { (reference) }\end{array}$ & 24,761 & 5.488 & $\begin{array}{c}1 \\
\text { (reference) }\end{array}$ \\
\hline $70-74$ & 14,284 & 4.42 & $\begin{array}{c}1.714 \\
(1.673-1.756)\end{array}$ & 83,714 & 28.29 & $\begin{array}{c}1.296 \\
(1.284-1.308)\end{array}$ & 44,346 & 14.322 & $\begin{array}{c}1.173 \\
(1.158-1.188)\end{array}$ & 29,601 & 9.197 & $\begin{array}{c}1.717 \\
(1.688-1.746)\end{array}$ \\
\hline$\geq 75$ & 17,148 & 7.903 & $\begin{array}{c}3.207 \\
(3.133-3.283)\end{array}$ & 77,633 & 40.008 & $\begin{array}{c}1.814 \\
(1.797-1.832)\end{array}$ & 33,207 & 15.924 & $\begin{array}{c}1.283 \\
(1.265-1.3)\end{array}$ & 39,400 & 18.282 & $\begin{array}{c}3.562 \\
(3.506-3.619)\end{array}$ \\
\hline \multicolumn{13}{|l|}{ Sex } \\
\hline Male & 24,803 & 5.245 & $\begin{array}{c}1 \\
\text { (reference) }\end{array}$ & 73,222 & 16.109 & $\begin{array}{c}1 \\
\text { (reference) }\end{array}$ & 36,888 & 7.926 & $\begin{array}{c}1 \\
\text { (reference) }\end{array}$ & 54,734 & 11.622 & $\begin{array}{c}1 \\
\text { (reference) }\end{array}$ \\
\hline Female & 18,590 & 3.577 & $\begin{array}{c}0.638 \\
(0.626-0.651)\end{array}$ & 178,979 & 39.17 & $\begin{array}{c}2.392 \\
(2.371-2.412)\end{array}$ & 92,974 & 19.04 & $\begin{array}{c}2.374 \\
(2.347-2.404)\end{array}$ & 39,028 & 7.54 & $\begin{array}{c}0.607 \\
(0.599-0.615)\end{array}$ \\
\hline
\end{tabular}

Results are presented as IR per 1,000 person-years for the follow-up period as analyzed by Cox hazard regression analysis adjusted for age and sex.

IR, incidence rate; HR, hazard ratio; CI, confidence interval.

Table 3. Incidence rates of geriatric syndromes and adjusted hazard ratios of each variable

\begin{tabular}{|c|c|c|c|c|c|c|c|c|}
\hline \multirow[b]{2}{*}{ Variable } & \multicolumn{2}{|c|}{ Fall-related fracture } & \multicolumn{2}{|r|}{ Delirium } & \multicolumn{2}{|r|}{ Incontinence } & \multicolumn{2}{|r|}{$\begin{array}{l}\text { Pressure ulcer } \\
\end{array}$} \\
\hline & $\begin{array}{l}\text { IR (per } \\
1000)\end{array}$ & HR $(95 \%$ CI) & $\begin{array}{l}\text { IR (per } \\
1000 \text { ) }\end{array}$ & $\mathrm{HR}(95 \% \mathrm{CI})$ & $\begin{array}{c}\text { IR (per } \\
1000)\end{array}$ & HR $(95 \%$ CI) & $\begin{array}{l}\text { IR (per } \\
1000)\end{array}$ & HR $(95 \%$ CI) \\
\hline Rural & 4.43087 & $0.999(0.98-1.019)$ & 29.2327 & $1.113(1.104-1.122)$ & 14.2791 & $1.102(1.09-1.115)$ & 9.94849 & $1.095(1.08-1.11)$ \\
\hline Smoking & 6.40744 & $1.47(1.432-1.508)$ & 21.5354 & $1.234(1.216-1.251)$ & 8.6353 & $0.943(0.923-0.963)$ & 13.1797 & 1.354 (1.33-1.379) \\
\hline \multicolumn{9}{|l|}{ Alcohol intake } \\
\hline Nondrinker & 4.3041 & 1 (reference) & 30.7318 & 1 (reference) & 15.2202 & 1 (reference) & 9.4609 & 1 (reference) \\
\hline$\leq 2 / \mathrm{wk}$ & 4.0377 & $0.864(0.84-0.889)$ & 18.5573 & $0.925(0.913-0.938)$ & 9.5696 & $0.914(0.897-0.931)$ & 8.386 & $0.805(0.789-0.821)$ \\
\hline$\geq 3 / \mathrm{wk}$ & 5.4013 & $1.05(1.018-1.083)$ & 19.3029 & $1.13(1.112-1.149)$ & 7.798 & $0.876(0.854-0.898)$ & 11.2578 & $0.967(0.946-0.988)$ \\
\hline Physical activity & 3.71191 & $0.802(0.786-0.819)$ & 21.9786 & $0.827(0.82-0.834)$ & 11.9597 & $0.934(0.923-0.945)$ & 7.6806 & $0.754(0.743 \mathrm{v} 0.764)$ \\
\hline \multicolumn{9}{|l|}{$\mathrm{BMI}\left(\mathrm{kg} / \mathrm{m}^{2}\right)$} \\
\hline$<18.5$ & 6.61419 & $1.201(1.151-1.251)$ & 41.4845 & $1.3(1.277-1.323)$ & 11.5816 & $0.95(0.92-0.98)$ & 17.8456 & $1.452(1.414-1.489)$ \\
\hline $18.5-23$ & 4.94884 & 1 (reference) & 29.9695 & 1 (reference) & 11.8732 & 1 (reference) & 10.798 & 1 (reference) \\
\hline $23-25$ & 4.02681 & $0.881(0.86-0.903)$ & 25.5081 & $0.857(0.848-0.866)$ & 13.2322 & $1.106(1.09-1.123)$ & 8.4494 & $0.859(0.844-0.873)$ \\
\hline$\geq 25$ & 3.74862 & $0.883(0.863-0.904)$ & 25.3063 & $0.793(0.786-0.801)$ & 16.0911 & $1.258(1.241-1.274)$ & 7.8843 & $0.872(0.858-0.886)$ \\
\hline Diabetes mellitus & 5.81724 & $1.556(1.525-1.587)$ & 28.4285 & 1.035 (1.025-1.044) & 16.6059 & $1.305(1.289-1.321)$ & 13.2983 & $1.691(1.668-1.714)$ \\
\hline Hypertension & 4.70143 & $1.163(1.14-1.186)$ & 27.4896 & $0.922(0.915-0.93)$ & 14.4449 & $1.117(1.105-1.13)$ & 10.3327 & $1.198(1.182-1.214)$ \\
\hline Dyslipidemia & 4.38582 & $1.126(1.101-1.151)$ & 28.5867 & $0.928(0.92-0.937)$ & 15.9371 & $1.095(1.082-1.108)$ & 9.00347 & $1.063(1.047-1.079)$ \\
\hline Stroke & 6.79938 & $1.624(1.587-1.663)$ & 32.9003 & $1.175(1.162-1.188)$ & 18.1288 & $1.374(1.354-1.394)$ & 15.5513 & $1.72(1.694-1.747)$ \\
\hline Dementia & 12.8166 & $2.74(2.628-2.852)$ & 42.2003 & $1.321(1.29-1.352)$ & 22.1593 & $1.506(1.46-1.554)$ & 30.2611 & $2.914(2.832-2.988)$ \\
\hline Heart disease & 7.11197 & $1.624(1.576-1.674)$ & 34.3726 & $1.14(1.124-1.157)$ & 17.9685 & $1.263(1.238-1.287)$ & 15.8643 & $1.655(1.621-1.689)$ \\
\hline Pulmonary disease & 5.71706 & $1.369(1.34-1.398)$ & 31.5559 & $1.199(1.188-1.21)$ & 16.5451 & $1.32(1.303-1.336)$ & 12.0687 & $1.305(1.286-1.324)$ \\
\hline Liver disease & 6.16519 & 1.505 (1.441-1.572) & 30.3325 & $1.192(1.168-1.217)$ & 15.9556 & $1.258(1.224-1.293)$ & 13.816 & $1.566(1.521-1.612)$ \\
\hline Kidney disease & 11.6004 & $2.585(2.426-2.75)$ & 31.0511 & $1.212(1.164-1.26)$ & 13.8096 & $1.106(1.043-1.171)$ & 22.1746 & $2.23(2.13-2.333)$ \\
\hline Cancer & 6.68994 & $1.501(1.451-1.554)$ & 28.0938 & $1.182(1.162-1.203)$ & 15.832 & $1.364(1.333-1.395)$ & 15.9736 & $1.65(1.613-1.687)$ \\
\hline Osteoporosis & 4.4398 & $1.237(1.204-1.27)$ & 43.4631 & $1.326(1.314-1.339)$ & 21.8231 & $1.374(1.357-1.392)$ & 9.34199 & $1.219(1.197-1.241)$ \\
\hline Urinary tract infection & 5.03939 & $1.292(1.253-1.332)$ & 35.0633 & $1.12(1.106-1.133)$ & 29.3354 & $2.122(2.092-2.151)$ & 11.1301 & $1.336(1.309-1.364)$ \\
\hline Pneumonia & 7.05029 & $1.571(1.514-1.629)$ & 34.6964 & $1.274(1.252-1.296)$ & 16.4339 & $1.244(1.214-1.274)$ & 16.3654 & $1.664(1.624-1.705)$ \\
\hline Tuberculosis & 6.8133 & $1.546(1.472-1.622)$ & 32.7637 & $1.235(1.207-1.264)$ & 16.7265 & $1.289(1.249-1.33)$ & 15.2872 & $1.588(1.537-1.64)$ \\
\hline Polypharmacy & 6.84658 & $1.638(1.595-1.681)$ & 29.0699 & $1.062(1.048-1.076)$ & 16.5026 & $1.261(1.239-1.282)$ & 15.3419 & $1.692(1.662-1.722)$ \\
\hline Major surgery within 6 mo & 5.32153 & $1.179(1.123-1.237)$ & 33.2910 & $1.142(1.119-1.165)$ & 17.7605 & $1.264(1.229-1.298)$ & 11.3334 & $1.151(1.113-1.190)$ \\
\hline
\end{tabular}


differences, previous studies reported that women are more frail, functionally disabled, and vulnerable to geriatric syndromes than men, and while this study adds higher risk of delirium and incontinence in women, our results regarding fall-related fractures and pressure ulcers differ from those in the literature $\mathrm{e}^{11-13)}$.

It has been reported that women are more vulnerable to falls and fall-related injuries owing to relatively smaller muscle mass compared with men, decline of physiological functions such as visual function and balance, and medication effects ${ }^{11,14)}$. However, most common fall-related injuries in women are mild bruises and contusions, and only approximately $5 \%$ require hospitalized treatment as most injuries do not result in severe damage ${ }^{15,16)}$. Considering the nature of this study, i.e., the use of the claims data, it is likely that only people who experienced falls leading to fractures would have sought medical care, and it is possible that men, who engage relatively more in outdoor activities ${ }^{17)}$, presented a high risk of serious fracture from walking. Therefore, consideration of where older people spend most of their time and daily life will be necessary in future studies. Different results have been reported for different sexes regarding pressure ulcers. While some studies report female sex to be a risk factor of pressure ulcers, some report that older men have higher risk of pressure ulcers than older women ${ }^{4,18,19)}$.

Among comorbidities, dementia especially was associated with a higher risk of fall-related fractures, delirium, incontinence, and pressure ulcers than other diseases. Therefore, dementia must be considered as an important risk factor of geriatric syndromes and managed with more caution. Furthermore, chronic kidney diseases were associated with a higher risk of fractures and pressure ulcers. Lifestyle also affects the incidence of geriatric syndrome. Smoking and alcohol intake are common risk factors of many diseases, with differences in the risks for different geriatric syndromes. Exercise reduced the risk of fall-related fractures, delirium, incontinence, and pressure ulcers, whereas smoking increased the risks of fall-related fractures, delirium, and pressure ulcers while decreasing the risk of incontinence. It has been reported that smoking increases the risk of incontinence in women through an antiestrogenic effect and interference with collagen synthesis, but some report the correlation to be statistically not significant ${ }^{20-22}$. A study involving Japanese men reported a lower risk of incontinence in past smokers and a higher risk in current smokers compared with those who never smoked, but the results were not statistically significant ${ }^{23}$. Parity has a considerable effect on incontinence in women ${ }^{24)}$, but it was not analyzed in this study. It is possible that female smokers have a decreased possibility of conception and delivery, which may lead to a decreased rate of urinary incontinence.

Intake of alcohol twice a week was associated with a lower risk of fall-related fractures, delirium, incontinence, and pressure ulcers, while alcohol intake three times a week was associated with a higher risk of fall-related fractures and delirium and a lower risk of incontinence and pressure ulcers compared with those who do not drink at all. Drinking alcohol is often related to socializing activities, which may have a negative association with incontinence and pressure ulcers ${ }^{25,26)}$.

There have been a number of studies on the correlation between drinking and incontinence. A large prospective cohort study in the United Kingdom reported a lower incidence of incontinence in men who drink beer than in those who do not or barely drink alcohol ${ }^{23}$. A study of Japanese men over middle age inclusive of older people reported a lower risk of incontinence in the groups who drank 0.1-32 g per day and even more than $32 \mathrm{~g}$ per day than in the groups who do not or barely drink alcohol ${ }^{27}$. These studies argued that alcohol has protective effects against incontinence in relation to the urethra and contractile property of the detrusor muscle ${ }^{23)}$. Other studies argued that alcohol induces transient changes in circulating testosterone and estrogen levels, which may decrease the contractile power of detrusor muscle by inhibiting calcium influx through the calcium channels in the membranes of smooth muscles ${ }^{28,29)}$. However, excessive drinking nullifies such effects owing to diuretic effects. The correlation between drinking and incontinence in the literature has been inconsistent in women as opposed to men. Some studies report a high risk of incontinence in women who drink alcohol, while a longitudinal study in the United Kingdom reported no effects of alcohol on incontinence regardless of the incontinence type. Other cross-sectional studies reported no significant association between alcohol and incontinence $e^{20,22,30)}$.

The effects of alcohol on pressure ulcers have also been studied. Clinical evidence and previous animal studies suggest that alcohol disrupts the wound healing process ${ }^{31)}$, inhibits wound closure and collagen production, and increases the frequency of infection ${ }^{32)}$. Therefore, it affects not only the risk of pressure ulcers but also the recovery. However, the effects of alcohol differ with regard to acute or chronic conditions and the amount and duration of exposure ${ }^{33)}$, which may explain results showing no correlation between alcohol and pressure ulcers in previous studies ${ }^{25,26)}$. The results of our study present a negative correlation between alcohol and pressure ulcers. However, it is difficult to conclude that there is an association with risk because there is not enough information on duration, amount, and type of exposure. Further, as already mentioned, alcohol intake is often associated with socializing activities, which may have a negative correlation with the incidence of pressure ulcers.

The group with lower BMI than the normal weight group presented an increased risk of fall-related fractures, delirium, and pressure ulcers. The risk of incontinence was lower in 
the lower BMl group than in the higher BMl group and increased with increasing weight. This is in agreement with previous reports that obesity is related to an increased risk of incontinence, especially stress incontinence, in women ${ }^{34,35)}$. Low body weight in older people reflects their nutritional state and subsequent risk of various geriatric syndromes. In particular, pressure ulcers often result from a bedridden state due to various diseases, and decreased sensation, poor nutritional health, and weight loss are reported to be risk factors ${ }^{36)}$

Geriatric syndromes were identified using diagnostic codes in this study, and therefore, the actual incidence may be underestimated or overestimated. After reviewing other studies that have used claims data, it was confirmed that analyses were based on the International Classification of Diseases (ICD) in common. We studied only four types of geriatric syndrome due to the limitation of only being able to use diagnostic codes to extract insurance claims data. For instance, the 'E' code (for accidental fall) or 'W' code (for trauma) for falls are rarely entered by clinicians, which raises the limitation of not being able to accurately confirm the incidence of falls ${ }^{377}$. Another issue in using claims data is that the analysis includes only patients who were treated for fall-related fracture, incontinence, and pressure ulcers. As patients with more severe symptoms may be more likely to seek treatment and receive a diagnosis of geriatric syndrome, this data limitation could lead to an underestimation $^{38,39)}$. Regarding delirium, the definition varies (Diagnostic and Statistical Manual or ICD) according to the criteria used, and the hypoactive form has been underdiagnosed ${ }^{40)}$. These limitations include the possibility of inaccurate diagnoses, coding inaccuracies, and missing data. Furthermore, as the results of health check-ups offered by the NHIS are limited, there may be more potential confounding factors not addressed in this study.

Results for known risk factors such as alcohol intake and smoking were contradictory, probably due to lack of information on other confounding variables.

Despite the shortcomings, this study analyzed data of a large cohort of older people in the NHIS database and identified the risk factors of geriatric syndromes.

In conclusion, this study verified that old age, history of major surgery within the past 6 months, and polypharmacy increase the risk of fall-related fractures, delirium, incontinence, and pressure ulcers. Low body weight was also associated with a high risk of fall-related fractures, delirium, and pressure ulcers. On the other hand, the risk of incontinence decreased with decreasing body weight and viceversa. Exercise was a preventive factor for all four types of geriatric syndromes studied. Acute and chronic comorbidities, especially dementia, increased the incidence of geriatric syndromes. Contradictory results were found with respect to alcohol intake and smoking, which require further study.

Conflicts of Interest Disclosures: The researchers claim no conflicts of interest.

\section{Acknowledgements}

This study was supported by grants from the Korea Geriatrics Society. The National Health Information Database was provided by the National Health Insurance Service (NHIS) of Korea. The authors would like to thank the National Health Insurance Service for their cooperation and the authors also thank KD Han for his advice on data analysis.

\section{Supplementary Material}

Supplementary Table 1 can be found via http://www.e-agmr. $\mathrm{org} / \mathrm{src} / \mathrm{sm} /$ agmr-21-123-s002.pdf.

\section{REFERENCES}

1. Korea statistical information system (KOSIS). Population structure by age and sex [Internet]. Daejeon (Korea): Statistics Korea; [updated 2016 Dec 12; cited 2017 May 7]. Available from: http:// www.index.go.kr/potal/main/EachDtlPageDetail.do?idx_cd=1010.

2. He W, Goodkind D, Kowal P. U.S. Census Bureau. International Population Reports, P95/16-1. An aging world: 2015. Washington, DC: U.S. Government Publishing Office; 2016.

3. Halter JB, Ouslander JG, Tinetti M, Studenski S, High KP, Asthana S. Hazzard's geriatric medicine and gerontology. 6th ed. New York: McGraw-Hill Prof Med/Tech; 2009.

4. Inouye SK, Studenski S, Tinetti ME, Kuchel GA. Geriatric syndromes: clinical, research, and policy implications of a core geriatric concept. J Am Geriatr Soc 2007;55:780-91.

5. Won CW, Yoo HJ, Yu SH, Kim CO, Dumlao LC, Dewiasty $\mathrm{E}$, et al. Lists of geriatric syndromes in the Asian-Pacific geriatric societies. Eur Geriatr Med 2013;4:335-8.

6. Kane RL, Shamliyan T, Talley K, Pacala J. The association between geriatric syndromes and survival. J Am Geriatr Soc 2012; 60:896-904.

7. Lee J, Lee JS, Park SH, Shin SA, Kim K. Cohort Profile: The National Health Insurance Service-National Sample Cohort (NHIS-NSC), South Korea. Int J Epidemiol 2017;46:e15.

8. Sundararajan V, Henderson T, Perry C, Muggivan A, Quan H, Ghali WA. New ICD-10 version of the Charlson comorbidity index predicted in-hospital mortality. J Clin Epidemiol 2004; 57:1288-94.

9. Main surgery statistical yearbook [Internet]. Wonju (Korea): National Health Insurance Service; 2016 Dec 5 [cited 2017 Jan 5]. Available from: http://www.nhis.or.kr/bbs7/boards/B0079? boardKey $=33 \&$ boardName $=$ B0079\&viewType $=$ generic\&ro ws $=10 \&$ sort $=$ sequence $\&$ order $=$ desc $\&$ pageNumber $=1 \&$ mess ageCategoryKey $=\&$ status $=$ \&period $=\&$ starPointdt $=$ \&enddt $=$ \&queryField=\&query $=\# 1$. 
10. Flacker JM. What is a geriatric syndrome anyway? J Am Geriatr Soc 2003;51:574-6.

11. Campbell AJ, Borrie MJ, Spears GF. Risk factors for falls in a community-based prospective study of people 70 years and older. J Gerontol 1989;44:M112-7.

12. Lim JY, Park WB, Oh MK, Kang EK, Paik NJ. Falls in a proportional region population in Korean elderly: incidence, consequences, and risk factors. J Korean Geriatr Soc 2010;14: 8-17.

13. Newman AB, Brach JS. Gender gap in longevity and disability in older persons. Epidemiol Rev 2001;23:343-50.

14. Tromp AM, Smit JH, Deeg DJ, Bouter LM, Lips P. Predictors for falls and fractures in the Longitudinal Aging Study Amsterdam. J Bone Miner Res 1998;13:1932-9.

15. Rubenstein LZ. Falls in older people: epidemiology, risk factors and strategies for prevention. Age Ageing 2006;35 Suppl 2: ii37-41.

16. Blake AJ, Morgan K, Bendall MJ, Dallosso H, Ebrahim SB, Arie $\mathrm{TH}$, et al. Falls by elderly people at home: prevalence and associated factors. Age Ageing 1988;17:365-72.

17. Lee E, Kim C. A survey of fractures and factor associated with falls in elderly patients. Journal Name 2003;5:182-92.

18. Baumgarten M, Margolis DJ, Localio AR, Kagan SH, Lowe RA, Kinosian B, et al. Pressure ulcers among elderly patients early in the hospital stay. J Gerontol A Biol Sci Med Sci 2006;61: 749-54.

19. de Souza DM, Santos VL. Risk factors for pressure ulcer development in institutionalized elderly. Rev Lat Am Enfermagem 2007; 15:958-64.

20. Hannestad YS, Rortveit G, Daltveit AK, Hunskaar S. Are smoking and other lifestyle factors associated with female urinary incontinence? The Norwegian EPINCONT Study. BJOG 2003;110: 247-54.

21. Bump RC, McClish DK. Cigarette smoking and urinary incontinence in women. Am J Obstet Gynecol 1992;167:1213-8.

22. Bortolotti A, Bernardini B, Colli E, Di Benedetto P, Giocoli Nacci G, Landoni $M$, et al. Prevalence and risk factors for urinary incontinence in Italy. Eur Urol 2000;37:30-5.

23. Dallosso HM, Matthews RJ, McGrother CW, Donaldson MM, Shaw C; Leicestershire MRC Incontinence Study Group. The association of diet and other lifestyle factors with the onset of overactive bladder: a longitudinal study in men. Public Health Nutr 2004;7:885-91.

24. Parazzini F, Chiaffarino F, Lavezzari M, Giambanco V; VIVA Study Group. Risk factors for stress, urge or mixed urinary incontinence in Italy. BJOG 2003;110:927-33.

25. Cakmak SK, Gül U, Ozer S, Yiğit Z, Gönü M. Risk factors for pressure ulcers. Adv Skin Wound Care 2009;22:412-5.

26. Tate DG, Forchheimer MB, Krause JS, Meade MA, Bombardier
$\mathrm{CH}$. Patterns of alcohol and substance use and abuse in persons with spinal cord injury: risk factors and correlates. Arch Phys Med Rehabil 2004;85:1837-47.

27. Lee AH, Hirayama F. Is alcohol consumption associated with male urinary incontinence? Low Urin Tract Symptoms 2011;3: 19-24.

28. Couwenbergs CJ. Acute effects of drinking beer or wine on the steroid hormones of healthy men. J Steroid Biochem 1988; $31(4 \mathrm{~A}): 467-73$.

29. Ida Y, Tsujimaru S, Nakamaura K, Shirao I, Mukasa H, Egami $\mathrm{H}$, et al. Effects of acute and repeated alcohol ingestion on hypothalamic-pituitary-gonadal and hypothalamic-pituitary-adrenal functioning in normal males. Drug Alcohol Depend 1992; 31:57-64.

30. Hsieh CH, Lee MS, Lee MC, Kuo TC, Hsu CS, Chang ST. Risk factors for urinary incontinence in Taiwanese women aged 20-59 years. Taiwan J Obstet Gynecol 2008;47:197-202.

31. Greiffenstein P, Molina PE. Alcohol-induced alterations on host defense after traumatic injury. J Trauma 2008;64:230-40.

32. Szabo G, Mandrekar P. A recent perspective on alcohol, immunity, and host defense. Alcohol Clin Exp Res 2009;33:220-32.

33. Guo S, Dipietro LA. Factors affecting wound healing. J Dent Res 2010;89:219-29.

34. Dwyer PL, Lee ET, Hay DM. Obesity and urinary incontinence in women. Br J Obstet Gynaecol 1988;95:91-6.

35. Moreno-Vecino B, Arija-Blázquez A, Pedrero-Chamizo R, Alcázar J, Gómez-Cabello A, Pérez-López FR, et al. Associations between obesity, physical fitness, and urinary incontinence in non-institutionalized postmenopausal women: The elderly EXERNET multi-center study. Maturitas 2015;82:208-14.

36. Horn SD, Buerhaus P, Bergstrom N, Smout RJ. RN staffing time and outcomes of long-stay nursing home residents: pressure ulcers and other adverse outcomes are less likely as RNs spend more time on direct patient care. Am J Nurs 2005;105: 58-70.

37. Langley J, Stephenson S, Thorpe C, Davie G. Accuracy of injury coding under ICD-9 for New Zealand public hospital discharges. Inj Prev 2006;12:58-61.

38. Oliphant SS, Wang L, Bunker CH, Lowder JL. Trends in stress urinary incontinence inpatient procedures in the United States, 1979-2004. Am J Obstet Gynecol 2009;200:521.e1-6.

39. Garber SL, Rintala DH. Pressure ulcers in veterans with spinal cord injury: a retrospective study. J Rehabil Res Dev 2003;40: 433-41.

40. Inouye SK, Leo-Summers L, Zhang Y, Bogardus ST Jr, Leslie DL, Agostini JV. A chart-based method for identification of delirium: validation compared with interviewer ratings using the confusion assessment method. J Am Geriatr Soc 2005;53:312-8. 